\title{
Optimal Control Applications in the Study of Production Management
}

\author{
L. Popescu, N. D. Militaru, O. M. Mituca
}

\section{Liviu Popescu*}

Department of Statistics and Economic Informatics Faculty of Economics and Business Administration, University of Craiova, Al. I. Cuza st. no. 13, Craiova 200585, Romania

*Corresponding author: liviunew@yahoo.com

\section{Nicolae Daniel Militaru}

University of Craiova

Al. I. Cuza st. no. 13, Craiova 200585, Romania

Postdoctoral researcher

militarunicolaedaniel@yahoo.com

\section{Ovidiu Mircea Mituca}

Academy of Economic Studies

Piata Romana, no. 6, Bucharest 010374, Romania

PhD student

mircea.mituca@gmail.com

\begin{abstract}
A mathematical model for an economic problem of production management is proposed. The continuous optimal control problem is solved, by using the Pontryagin Maximum Principle at the level of a new space, called Lie algebroid. The controllability of the economic system is studied by using Lie geometric methods and involves restrictions on the final stock quantities. Finally, a numerical application is given.

Keywords: optimal control, automatic control, controllability, production management, Pontryagin Maximum Principle, Lie algebra.
\end{abstract}

\section{Introduction}

The mathematical methods are applied successfully in optimal control theory with many applications in economics, business, finance or engineering. One of the motivations for this paper is the study of some type of Lagrangian systems with external constraints. These systems have important applications in many different areas as optimal control theory, econometrics, cybernetics, operational research (Caputo [3], Feichtinger \& Hartl [8], Liu [14], Sethi \& Thompson [22], Weber, [23] ) or automatic control (Caruntu et al. [4], Chitsaz et al. [6], Dubins [7]). Also, in control theory, the Lie 
geometric methods have been applied by many authors, see for example Agrachev \& Sachkov [1], Brocket [2], Isidori [10], Jurdjevic [11], LaValle [13], Popescu [20].

In this paper we propose a mathematical model for a problem of production management, using the optimal control techniques. We find the optimal solution with minimum costs of production and storage applying the Pontrygin Maximum Principle (PMP) at the level of a new working space, called Lie algebroid, which is a holonomic distribution of the tangent space. Moreover, it is provided that the framework of Lie algebroids is more suitable than the tangent space in the study of control affine systems with holonomic distributions. The controllability of our economic system is studied by using Lie geometric methods and applying the Frobenius theorem. Such type of problems including inventory and production management are intensely studied. Thus, Chazal, Jouini \& Tahraoui [5] studied the production planning and inventories optimization, using a backward approach for the case of convex storage cost. Gayon, Vercraene \& Flapper [9] dealt with optimal control of a production inventory system with two disposal options and product returns. Maccini, Moore \& Schaller [15] studied the inventory behavior with permanent sales stocks. Olsson [18] presented some modelling techniques for base-stock inventory systems in the case of state dependent demand rates. Ortega \& Lin [19] gave a review on control theory applications to the production and inventory problems. Popescu [21] used the framework of a driftless control affine system in the study of inventory and production problems. The present paper is organized as follows. In the second section we present the known results about Lie geometric methods in optimal control theory for control affine systems, including the controllability problems in the case of holonomic distributions. In section three, that contains the novelty of the paper, we give an application of optimal control to a problem of production management and show that the Hamilton-Jacobi-Bellman equations, provided by PMP on cotangent space, lead to a system of differential equations, very difficult to solve. In order to find the optimal solution, we will use a different way, applying the PMP in the framework of Lie algebroids. Next, we present only the necessary notions about Lie algebroids (see Mackenzie [17] for more details) and the geometric viewpoint of the optimal control. Using the relation between the Hamiltonian functions on dual Lie algebroid and the cotangent space, we apply the PMP at the level of a Lie algebroid, which in this case is a distribution on the tangent bundle. Next, we prove that the distribution generated by the vector fields is integrable and it determines a foliation in the state space. It results that the proposed economic system is not controllable, in the sense that we can only manufacture a certain final quantity of products. In the last part of the section, we find the complete solution of the control system using the PMP on Lie algebroids. Finally, a numerical example is given.

\section{Theoretical basic of control affine systems}

Let us consider $M$ be a smooth and $n$-dimensional manifold. A continuous control system is given by the differential equations, depending on some parameters

$$
\frac{d x^{i}(t)}{d t}=f^{i}(x(t), u(t)),
$$

where $x \in M$ represents the state of the system and $u \in U \subset R^{m}$ represents the controls. Considering $x_{0}$ and $x_{1}$ two points of $M$ (two states of the system), then the optimal control problem consists of finding the optimal trajectories of control system that connect $x_{0}$ and $x_{1}$ and minimizing the cost

$$
\min \int_{0}^{T} L(x(t), u(t)) d t, \quad x(0)=x_{0}, x(T)=x_{1},
$$

where the function $L$ is the Lagrangian (energy, cost, time, distance, etc.). The most important and powerful tool for studying the optimal solutions in control theory is PMP. It generates the differential equations of first order, necessary for the optimal solutions. For each optimal trajectory, $c(t)=(x(t), u(t))$, it offers a lift on the cotangent space $(x(t), p(t))$ satisfying Hamilton-Jacobi-Bellman equations. The Hamiltonian function is given by $H(x, p, u)=\langle p, f(x, u)\rangle-L(x, u), p \in T^{*} M$, and it results that the maximization condition with respect to $u$, given by

$$
H(x(t), p(t), u(t))=\max _{v} H(x(t), p(t), v),
$$


yields $\frac{\partial H}{\partial u}=0$ ( $H$ is assumed to be smooth with respect to $u$ ). The extreme trajectories must satisfy the following equations

$$
\dot{x}=\frac{\partial H}{\partial p}, \quad \dot{p}=-\frac{\partial H}{\partial x} .
$$

We recall that a control affine system has the form [2]

$$
\dot{x}=X_{0}(x)+\sum_{i=1}^{m} u^{i} X_{i}(x),
$$

where $x=\left(x^{1}, \ldots, x^{n}\right)$ represent the local coordinates on the manifold $M, u(t)=\left(u^{1}(t), \ldots, u^{m}(t)\right)$ $\in U \subset R^{m}, m \leq n$ and $X_{0}, X_{1} \ldots X_{m}$ are smooth vector fields on $M$. Usually, $X_{0}$ is called the drift vector field which describes the dynamics of the affine system in the absence of control. The vector fields $X_{i}, i=\overline{1, m}$ are called the input vector fields and the function $u(t)$ represents the control. We can say that the system is controllable if for any two states $x_{0}$ and $x_{1}$ of the system, there exists a finite time $T$ and a control $u:[0, T] \rightarrow U$ such that for $x(t)$ with $x(0)=x_{0}$, we have $x(T)=x_{1}$. In other words, the system is controllable if for any two states $x_{0}, x_{1}$, there exists a solution curve of the system (2) which connects $x_{0}$ to $x_{1}$. Controllability is the ability to move a system from a given initial state to any final state, in finite time, using some available controls. A distribution $\Delta$ on $M$ is an application which assigns for every point in $M$ a subspace of the tangent space at this point. The distribution $\Delta$ is called locally finitely generated if there is a set of vector fields $\left\{X_{i}\right\}_{i=\overline{1, m}}$ (called local generators of $\Delta$ ) which spans $\Delta$, i.e. $\Delta(x)=\operatorname{span}\left\{X_{1}(x), \ldots, X_{m}(x)\right\} \subset T_{x} M$. The distribution $\Delta$ has dimension $k$ if $\operatorname{dim} \Delta(x)=k$, for all points $x$ in $M$. The Lie bracket of two vector fields is given by $\left[X_{i}, X_{j}\right](x)=\frac{\partial X_{j}}{\partial x}(x) X_{i}(x)-\frac{\partial X_{i}}{\partial x}(x) X_{j}(x),\left(\frac{\partial X_{j}}{\partial x}\right.$ is the Jacobian matrix of $\left.X_{j}\right)$. A distribution $\Delta$ on $M$ is said to be involutive if, for any $x \in M$ and $X_{i}(x), X_{j}(x) \in \Delta(x)$, we have $\left[X_{i}, X_{j}\right](x) \in \Delta(x)$. If the involutive distribution is generated by vector fields $\left\{X_{i}\right\}_{i=\overline{1, m}}$ then it results

$$
\left[X_{i}, X_{j}\right](x)=\sum_{k=1}^{m} L_{i j}^{k}(x) X_{k}(x)
$$

Therefore, we can say that each Lie bracket is expressed as a linear combination of the system vector fields. We recall that a foliation $\left\{S_{\alpha}\right\}_{\alpha \in A}$ of $M$ is a partition of the space $M=\cup S_{\alpha}$ of $M$ in disjoint and connected submanifolds $S_{\alpha}$, called leaves. A distribution $\Delta$ of constant dimension on $M$ is called holonomic (integrable) if there exists a foliation $\left\{S_{\alpha}\right\}_{\alpha \in A}$ on $M$ whose tangent bundle is $\Delta$, that is $T_{x} S=\Delta(x)$, where $S$ is the leaf passing through $x$. Frobenius's theorem says that if the distribution $\Delta$ has constant dimension, then $\Delta$ is integrable if and only if $\Delta$ is involutive. The presence of the drift $X_{0}$ in the study of control affine systems complicates the question of controllability. In the following we deal with the driftless control affine system in the form

$$
\dot{x}=\sum_{i=1}^{m} u^{i} X_{i}(x) .
$$

It is assumed that the vector fields $X_{i}, i=\overline{1, m}$, generate a distribution $\Delta$ on the connected manifold $M$ so that the rank of $\Delta$ is constant. If the distribution $\Delta=\operatorname{span}\left\{X_{1}, X_{2}, \ldots, X_{m}\right\}$ is holonomic with constant rank, then $\left[X_{i}, X_{j}\right] \in \Delta$ for every $i \neq j$ and the system is not controllable. Using the Frobenius theorem it results that the distribution $\Delta$ is holonomic and determines a foliation on $M$. Thus, two points can be joined by a trajectory of the system if and only if they are situated on the same leaf of the foliation.

\section{Production management problem}

We consider that a company can manufacture $n$ types of products, denoted $P_{1}, P_{2}, \ldots, P_{n}$. In a certain period of time $T$ (fixed) it must produce a certain amount $\left(s_{1}, s_{2}, \ldots, s_{n}\right)$ of each type of product. The quantity of $P_{n}$ product depends on the quantities of products $P_{1}, P_{2}, \ldots, P_{n-1}$ by a given law. We know that the unit storage costs of holding inventory are given by constants $\left(\beta_{1}, \beta_{2}, \ldots, \beta_{n}\right)$ for each 
product. Also, we assume that the unit production costs increase linearly with the production level and the cost of production operations for $P_{n}$ are considered negligible $\left(P_{n}\right.$ is a final product packed and unassembled). We are looking for a plan of production for filling the order at the specified delivery data at minimum cost. The case of a single product is studied by Kamien and Schwartz [12]. We consider $x^{i}=x^{i}(t)$ the inventory accumulated by time $t$. The inventory level is the cumulated past production $p^{i}=p^{i}(t)$ and considering $x^{i}(0)=0$, we have

$$
x^{i}(t)=\int_{0}^{t} p^{i}(s) d s
$$

Hence the rate of change of inventory level $\dot{x}^{i}$ is the production and we have $\dot{x}^{i}=p^{i}$. The unit production $\operatorname{costs} c_{i}$ increase linearly with the production level, i.e $c_{i}=\alpha_{i} p^{i}$, where $\alpha_{1}, \ldots, \alpha_{n-1} \in(0,1)$ are positive constants and we have that the total cost of production is

$$
c_{1} p^{1}+\ldots+c_{n-1} p^{n-1}=\sum_{i=1}^{n-1} \alpha_{i}\left(p^{i}\right)^{2}=\sum_{i=1}^{n-1} \alpha_{i}\left(\dot{x}^{i}\right)^{2} .
$$

We obtain that the total cost (including storage), at time $t$ is

$$
\sum_{i=1}^{n-1} \alpha_{i}\left(\dot{x}^{i}\right)^{2}+\sum_{i=1}^{n} \beta_{i} x^{i} .
$$

Considering, $\dot{x}^{i}=u^{i}, i=\overline{1, n-1}$, the control variables and assuming that the rate of change of inventory for final product $P_{n}$ is given by the law

$$
\dot{x}^{n}(t)=u^{1} \frac{x^{1}(t)}{k_{1}}+\ldots+u^{n-1} \frac{x^{n-1}(t)}{k_{n-1}},
$$

where $k_{i}>0, i=\overline{1, n-1}$, we obtain the following optimal control problem

$$
\begin{aligned}
& \left\{\begin{array}{l}
\dot{x}^{1}=u^{1} \\
\dot{x}^{2}=u^{2} \\
\ldots . . \\
\dot{x}^{n-1}=u^{n-1} \\
\dot{x}^{n}=u^{1} \frac{x^{1}}{k_{1}}+\ldots+u^{n-1} \frac{x^{n-1}}{k_{n-1}} \\
x^{i}(0)=0, \quad x^{i}(T)=s_{i}, \quad i=\overline{1, n} \\
u^{1}, \ldots, u^{n-1} \geq 0
\end{array}\right. \\
& \min \int_{0}^{T}\left(\alpha_{1}\left(u^{1}\right)^{2}+\ldots+\alpha_{n-1}\left(u^{n-1}\right)^{2}+\beta_{1} x^{1}+\ldots+\beta_{n} x^{n}\right) d t .
\end{aligned}
$$

We are looking for the optimal solutions starting from the point $(0,0, \ldots, 0)$ and $\left(s_{1}, s_{2}, \ldots s_{n}\right)$ as endpoint. The system can be written in the form (driftless control affine system):

$$
\begin{gathered}
\dot{x}=\sum_{i=1}^{n-1} u^{i} X_{i}, \quad x=\left(\begin{array}{c}
x^{1} \\
\vdots \\
x^{n}
\end{array}\right), X_{1}=\left(\begin{array}{c}
1 \\
0 \\
\vdots \\
\frac{x^{1}}{k_{1}}
\end{array}\right), \ldots, X_{n-1}=\left(\begin{array}{c}
0 \\
\vdots \\
1 \\
\frac{x^{n-1}}{k_{n-1}}
\end{array}\right) \in R_{+}^{n} \\
\min \int_{0}^{T} \mathcal{F}(u(t), x(t)) d t, \\
\mathcal{F}(u(t), x(t))=\alpha_{1}\left(u^{1}\right)^{2}+\ldots+\alpha_{n-1}\left(u^{n-1}\right)^{2}+\beta_{1} x^{1}+\ldots+\beta_{n} x^{n} .
\end{gathered}
$$

The distribution $\Delta=\operatorname{span}\left\{X_{1}, \ldots, X_{n-1}\right\}$ which is generated by the vector fields $X_{1}, \ldots, X_{n-1}$ has constant dimension, $\operatorname{dim} \Delta(x)=n-1$, for all $x \in R^{n}$. The vector fields are given by

$$
X_{1}=\frac{\partial}{\partial x^{1}}+\frac{x^{1}}{k_{1}} \frac{\partial}{\partial x^{n}}, \quad X_{2}=\frac{\partial}{\partial x^{2}}+\frac{x^{2}}{k_{2}} \frac{\partial}{\partial x^{n}}, \ldots, X_{n-1}=\frac{\partial}{\partial x^{n-1}}+\frac{x^{n-1}}{k_{n-1}} \frac{\partial}{\partial x^{n}} .
$$


The Lie bracket are

$$
\left[X_{i}, X_{j}\right]=\left[\frac{\partial}{\partial x^{i}}+\frac{x^{i}}{k_{i}} \frac{\partial}{\partial x^{n}}, \frac{\partial}{\partial x^{j}}+\frac{x^{j}}{k_{j}} \frac{\partial}{\partial x^{n}}\right]=0, \quad i, j \in \overline{1, n-1}
$$

and it results that the associated distribution $\Delta=\operatorname{span}\left\{X_{1}, \ldots, X_{n-1}\right\}$ is holonomic and has the constant rank $n-1$. Using the Frobenius theorem, it results that the system is not controllable, in the sense that we cannot reach any final stock quantity. Moreover, from the system (4) we obtain

$$
\dot{x}^{n}=\frac{\dot{x}^{1} x^{1}}{k_{1}}+\ldots+\frac{\dot{x}^{n-1} x^{n-1}}{k_{n-1}},
$$

which yields, by integration

$$
x^{n}=\frac{\left(x^{1}\right)^{2}}{2 k_{1}}+\ldots+\frac{\left(x^{n-1}\right)^{2}}{2 k_{n-1}}+c .
$$

( $c$ is a constant) and it results that $\Delta$ determines a foliation on $R_{+}^{n}$ given by the hipersurfaces (6). From the initial condition $x^{i}(0)=0$ it results $c=0$ and using that $x^{i}(T)=s_{i}$ we have that the system is controllable (the problem has the solution) if and only if the final amounts satisfy the condition

$$
s_{n}=\frac{s_{1}^{2}}{2 k_{1}}+\ldots+\frac{s_{n-1}^{2}}{2 k_{n-1}} .
$$

To solve the optimal control problem we can use the Pontryagin Maximum Principle on the cotangent space. We get the Hamiltonian

$$
\begin{gathered}
H(u, x, p)=\sum_{i=1}^{n} p_{i} \dot{x}^{i}-\mathcal{F}=p_{1} u^{1}+p_{2} u^{2}+\ldots+p_{n}\left(u^{1} \frac{x^{1}}{k_{1}}+\ldots+u^{n-1} \frac{x^{n-1}}{k_{n-1}}\right) \\
-\alpha_{1}\left(u^{1}\right)^{2}-\ldots-\alpha_{n-1}\left(u^{n-1}\right)^{2}-\beta_{1} x^{1}-\ldots-\beta_{n} x^{n}
\end{gathered}
$$

The conditions $\frac{\partial H}{\partial u^{i}}=0$ lead to the following formulas of control variables

$$
u^{i}=\frac{p_{i} k_{i}+p_{n} x^{i}}{2 \alpha_{i} k_{i}}
$$

which replaced into the expression of the Hamiltonian function leads to

$$
H=\sum_{i=1}^{n-1} \frac{\left(p_{i} k_{i}+p_{n} x^{i}\right)^{2}}{4 \alpha_{i} k_{i}^{2}}-\beta_{1} x^{1}-\ldots-\beta_{n} x^{n} .
$$

Using the Hamilton-Jacobi-Bellman equations (1) we obtain a very complicated system of differential equations. Therefore, we will use a different way, involving the framework of Lie algebroids.

\subsection{Lie algebroids}

We consider $M$ a real, $C^{\infty}$-differentiable, $n$-dimensional manifold and $T_{x} M$ its tangent space in the point $x \in M$. The tangent bundle of $M$ is usually noted $\left(T M, \pi_{M}, M\right)$, where $\pi_{M}$ is the canonical projection map $\pi_{M}: T M \rightarrow M$ applying a tangent vector $X(x) \in T_{x} M \subset T M$ to the base point $x \in M$. A vector bundle is a triple denoted $(E, \pi, M)$ with $E$ the total space, $M$ the base space and the map $\pi: E \rightarrow M$ is a surjective submersion. Using Mackenzie [17] we have:

Definition 1. A Lie algebroid is given by $\left(E,[\cdot, \cdot]_{E}, \sigma\right)$, where $(E, \pi, M)$ is a vector bundle of rank $m$ over the manifold $M$, satisfying the following conditions:

i) $C^{\infty}(M)$-module of sections $\Gamma(E)$ is endowed with a Lie algebra structure $[\cdot, \cdot]_{E}$.

ii) The map $\sigma: E \rightarrow T M$ (called the anchor) induces a Lie algebra homomorphism from the Lie algebra of sections $\left(\Gamma(E),[\cdot, \cdot]_{E}\right)$ to the Lie algebra of vector fields $(\succ(M),[\cdot, \cdot])$ and verifies the Leibniz rule

$$
\left[s_{1}, f s_{2}\right]_{E}=f\left[s_{1}, s_{2}\right]_{E}+\left(\sigma\left(s_{1}\right) f\right) s_{2}, \forall s_{1}, s_{2} \in \Gamma(E), f \in C^{\infty}(M) .
$$


Considering the local coordinates $\left(x^{i}\right)$ on an open subset $U \subset M$ and a local basis $\left\{s_{\alpha}\right\}$ of the sections of the bundle $\pi^{-1}(U) \rightarrow U$, these generate the local coordinates $\left(x^{i}, y^{\alpha}\right)$ on $E$. The local functions $\sigma_{\alpha}^{i}(x), L_{\alpha \beta}^{\gamma}(x)$ on the base $M$ given by following relations

$$
\sigma\left(s_{\alpha}\right)=\sigma_{\alpha}^{i} \frac{\partial}{\partial x^{i}}, \quad\left[s_{\alpha}, s_{\beta}\right]_{E}=L_{\alpha \beta}^{\gamma} s_{\gamma}, \quad i=\overline{1, n}, \quad \alpha, \beta, \gamma=\overline{1, m}
$$

are called the structure functions of Lie algebroids. We recall that a control system on the Lie algebroid $\left(E,[\cdot, \cdot]_{E}, \sigma\right)$ (see Martinez [16]) with the control space $\tau: A \rightarrow M$ is given by a section $\rho$ of $E$ along $\tau$. A trajectory of the control system $\rho$ is an integral curve of the vector field $\sigma(\rho)$. Considering the cost function $\mathcal{L} \in C^{\infty}(A)$, we must minimize the integral of $\mathcal{L}$ over the set of those system trajectories which satisfy certain optimal conditions. The Hamiltonian function $\mathcal{H}$ is given by $\mathcal{H}(\mu, u)=\langle\mu, \rho(u)\rangle-\mathcal{L}(u)$ and the critical trajectories are given by Hamilton-Jacobi equations on Lie algebroids (Martinez [16])

$$
\frac{d x^{i}}{d t}=\sigma_{\alpha}^{i} \frac{\partial \mathcal{H}}{\partial \mu_{\alpha}}, \quad \frac{d \mu_{\alpha}}{d t}=-\sigma_{\alpha}^{i} \frac{\partial \mathcal{H}}{\partial x^{i}}-\mu_{\gamma} L_{\alpha \beta}^{\gamma} \frac{\partial \mathcal{H}}{\partial \mu_{\beta}} .
$$

with $\frac{\partial \mathcal{H}}{\partial u^{A}}=0$. Using [20] we have:

Remark 3.1. The connection between the Hamiltonian function $H$ on the dual space $T^{*} M$ and the Hamiltonian function $\mathcal{H}$ on the dual Lie algebroid $E^{*}$ has the form

$$
H(p)=\mathcal{H}\left(\sigma^{\star}(p)\right), \quad \mu=\sigma^{\star}(p), \quad p \in T_{x}^{*} M, \quad \mu \in E_{x}^{*} .
$$

which locally yields

$$
\mu_{\alpha}=\sigma_{\alpha}^{* i} p_{i}
$$

and the Hamiltonian $H(p)$ is degenerate on the subset $K e r \sigma^{\star} \subset T^{*} M$.

\subsection{Solution of production management problem}

We will solve the problem of production management using the framework of Lie algebroids. Thus, we consider the space $E=\Delta$ (holonomic distribution with constant rank), the anchor map $\sigma: E \rightarrow T M$ is given by inclusion and $[,]_{E}$ is the induced Lie bracket. The anchor $\sigma$ has the components

$$
\sigma_{\alpha}^{i}=\left(\begin{array}{cccc}
1 & 0 & \ldots & 0 \\
0 & 1 & \ldots & 0 \\
\vdots & \vdots & & 0 \\
0 & 0 & \ldots & 1 \\
\frac{x^{1}}{k_{1}} & \frac{x^{2}}{k_{2}} & \ldots & \frac{x^{n-1}}{k_{n-1}}
\end{array}\right),
$$

and we get the Lagrangian function $\mathcal{L}$ on $E$ given by

$$
\mathcal{L}=\alpha_{1}\left(u^{1}\right)^{2}+\ldots+\alpha_{n-1}\left(u^{n-1}\right)^{2}+\beta_{1} x^{1}+\ldots+\beta_{n} x^{n},
$$

which is regular, because $\operatorname{det}\left(\frac{\partial^{2} L}{\partial u^{i} \partial u^{j}}\right) \neq 0$. Next, we use the Legendre transformation in order to find the Hamiltonian function on dual Lie algebroid.

Theorem 2. The Hamiltonian function on dual Lie algebroid $E^{*}$ has the following expression

$$
\mathcal{H}(x, \mu)=\sum_{i=1}^{n-1} \frac{\mu_{i}^{2}}{4 \alpha_{i}}-\beta_{1} x^{1}-\ldots-\beta_{n} x^{n} .
$$

Proof. The Legendre transformation from $E$ to $E^{*}$ induced by the regular Lagrangian $\mathcal{L}$ has the form

$$
(x, u) \rightarrow(x, \mu), \quad \mu_{i}=\Phi_{i}(x, u)=\frac{\partial \mathcal{L}}{\partial u^{i}}=2 \alpha_{i} u^{i},
$$


and the Hamiltonian function is given by $\mathcal{H}(x, \mu)=\mu \Phi^{-1}(x, \mu)-\mathcal{L}\left(x, \Phi^{-1}(x, \mu)\right)$. In $(x, u)$ coordinates we get

$$
\mathcal{H}=u^{i} \frac{\partial \mathcal{L}}{\partial u^{i}}-\mathcal{L}=\alpha_{1}\left(u^{1}\right)^{2}+\ldots+\alpha_{n-1}\left(u^{n-1}\right)^{2}-\beta_{1} x^{1}-\ldots-\beta_{n} x^{n},
$$

and using the relations $u^{i}=\frac{\mu_{i}}{2 \alpha_{i}}$, we find (12).

Using the relation (11) we can find the Hamiltonian function $H$ on $T^{*} M$ in the form $H(x, p)=$ $\mathcal{H}(\mu), \mu=\sigma^{\star}(p)$, with

$$
\left(\begin{array}{c}
\mu_{1} \\
\vdots \\
\mu_{n-1}
\end{array}\right)=\left(\begin{array}{ccccc}
1 & 0 & \ldots & 0 & \frac{x^{1}}{k_{1}} \\
0 & 1 & \ldots & 0 & \frac{x^{2}}{k_{2}} \\
\vdots & \vdots & & \vdots & \vdots \\
0 & 0 & \ldots & 1 & \frac{x^{n-1}}{k_{n-1}}
\end{array}\right)\left(\begin{array}{c}
p_{1} \\
\vdots \\
p_{n-1} \\
p_{n}
\end{array}\right) .
$$

We get that

$$
\mu_{i}=\frac{p_{i} k_{i}+p_{n} x^{i}}{k_{i}}
$$

and it results the Hamiltonian on the dual space, given in relation (8).

Theorem 3. The optimal solution of production management problem is given by

$$
\begin{gathered}
x^{i}(t)=a_{i_{1}} e^{\sqrt{\frac{\beta_{n}}{2 \alpha_{i} k_{i}}} t}+a_{i_{2}} e^{-\sqrt{\frac{\beta_{n}}{2 \alpha_{i} k_{i}}} t}-\frac{\beta_{i} k_{i}}{\beta_{n}}, \quad i=\overline{1, n-1}, \\
x^{n}(t)=\frac{\left(x^{1}(t)\right)^{2}}{2 k_{1}}+\ldots+\frac{\left(x^{n-1}(t)\right)^{2}}{2 k_{n-1}},
\end{gathered}
$$

where

$$
a_{i_{1}}=\frac{1}{d_{i}+1} \frac{\beta_{i} k_{i}}{\beta_{n}}+\frac{d_{i} s_{i}}{d_{i}^{2}-1}, \quad a_{i_{2}}=\frac{d_{i}}{d_{i}+1} \frac{\beta_{i} k_{i}}{\beta_{n}}-\frac{d_{i} s_{i}}{d_{i}^{2}-1}, \quad d_{i}=e^{\sqrt{\frac{\beta_{n}}{2 \alpha_{i} k_{i}}} T},
$$

and control variables

$$
u^{i}(t)=\sqrt{\frac{\beta_{n}}{2 \alpha_{i} k_{i}}}\left(a_{i_{1}} e^{\sqrt{\frac{\beta_{n}}{2 \alpha_{i} k_{i}}} t}-a_{i_{2}} e^{-\sqrt{\frac{\beta_{n}}{2 \alpha_{i} k_{i}}} t}\right) .
$$

Proof. From the relation $\left[X_{\alpha}, X_{\beta}\right]=L_{\alpha \beta}^{\gamma} X_{\gamma}$ we obtain the components $L_{\alpha \beta}^{\gamma}=0$, while from (9) we deduce that

$$
\frac{d x^{i}}{d t}=\sigma_{\alpha}^{i} \frac{\partial \mathcal{H}}{\partial \mu_{\alpha}}, \quad \frac{d \mu_{\alpha}}{d t}=-\sigma_{\alpha}^{i} \frac{\partial \mathcal{H}}{\partial x^{i}},
$$

which leads to

$$
\left\{\begin{array}{l}
\dot{x}^{i}=\frac{\mu_{i}}{2 \alpha_{i}}, \quad i=\overline{1, n-1} \\
\dot{x}^{n}=\sum_{i=1}^{n-1} \frac{\mu_{i} x^{i}}{2 \alpha_{i} k_{i}} \\
\dot{\mu}_{i}=\beta_{i}+\frac{x^{i}}{k_{i}} \beta_{n}, \quad i=\overline{1, n-1}
\end{array}\right.
$$

We deduce, using (17)

$$
\ddot{x}^{i}=\frac{\dot{\mu}_{i}}{2 \alpha_{i}} \Rightarrow \ddot{x}^{i}=\frac{\beta_{n}}{2 \alpha_{i} k_{i}} x^{i}+\frac{\beta_{i}}{2 \alpha_{i}}, \quad i=\overline{1, n-1},
$$

which yields the linear nonhomogeneous second order differential equations. Next, considering the linear homogeneous differential equations

$$
\ddot{x}^{i}-\frac{\beta_{n}}{2 \alpha_{i} k_{i}} x^{i}=0,
$$


and the characteristic equation $\lambda^{2}-\frac{\beta_{n}}{2 \alpha_{i} k_{i}}=0$, we get the solutions $\lambda_{1,2}= \pm \sqrt{\frac{\beta_{n}}{2 \alpha_{i} k_{i}}}$. It results the general solutions of the homogeneous differential equations

$$
x^{i}(t)=a_{i_{1}} e^{\sqrt{\frac{\beta_{n}}{2 \alpha_{i} k_{i}}} t}+a_{i_{2}} e^{-\sqrt{\frac{\beta_{n}}{2 \alpha_{i} k_{i}}} t} .
$$

Also, we find the general solution of the nonhomogeneous second order differential equations given by (13). The solution is optimal because the Hamiltonian is a convex function. The constants $a_{i_{1}}, a_{i_{2}}$ can be found from the initial conditions $x^{i}(0)=0, x^{i}(T)=s_{i}$, which lead to the system

$$
\left\{\begin{array}{l}
a_{i_{1}}+a_{i_{2}}=\frac{\beta_{i} k_{i}}{\beta_{n}} \\
a_{i_{1}} d_{i}+\frac{1}{d_{i}} a_{i_{2}}=\frac{\beta_{i} k_{i}}{\beta_{n}}+s_{i}
\end{array}\right.
$$

where we denote $d_{i}=e^{\sqrt{\frac{\beta_{n} k_{i}}{2 \alpha_{i}}} \text { T }}>1$. From the above system, by direct computation, the coefficients (15) are obtained. Finally, using (4) we have $\dot{x}^{i}=u^{i}, i=\overline{1, n-1}$ and it results the control variables (16), which ends the proof.

Numerical application: We consider a numerical example for the case $n=3$ :

- fixed period of time is $T=1$;

- final stock quantities from products $P_{1}, P_{2}, P_{3}$ are $s_{1}=6, s_{2}=4, s_{3}=8$;

- storage costs are given by $\beta_{1}=4, \beta_{2}=2, \beta_{3}=4$;

- the coefficients are $\alpha_{1}=2 / 3, \alpha_{2}=1 / 2, k_{1}=3, k_{2}=4$.

The economic system is controllable, because

$$
s_{3}=\frac{s_{1}^{2}}{2 k_{1}}+\frac{s_{2}^{2}}{2 k_{2}}=8
$$

If $s_{3} \neq 8$, then the system is not controllable. Applying the formulas (13)-(16) the following optimal solution is obtained

$$
\begin{aligned}
x^{1}(t) & =3,3595786488 e^{t}-0,3595786488 e^{-t}-3 \\
x^{2}(t) & =2,2397190992 e^{t}-0,2397190992 e^{-t}-2, \\
x^{3}(t) & =\frac{\left(x^{1}(t)\right)^{2}}{6}+\frac{\left(x^{2}(t)\right)^{2}}{8}
\end{aligned}
$$

with control variables (production rates) given by

$$
\begin{aligned}
& u^{1}(t)=3,3595786488 e^{t}+0,3595786488 e^{-t}, \\
& u^{2}(t)=2,2397190992 e^{t}+0,2397190992 e^{-t} .
\end{aligned}
$$

\section{Conclusions and future work}

In this paper a mathematical model for a problem of production management is proposed. We have to find the optimal solution so that different products are manufactured in the required quantities and at a fixed date with minimum production and storage costs. The continuous optimal control problem is solved by using the Pontryagin Maximum Principle at the level of a new working space, called Lie algebroid, which is a holonomic distribution on tangent spaces. The controllability of our economic system is solved by using the Lie geometric methods and the properties of the Lie brackets for the vectors fields which generate the distribution. The system is controllable if and only if the final stock quantities satisfy a given law. Finally, a numerical application is presented. We have to remark that the optimal solution of this numerical example can be easily represented in three-dimensional space using mathematical software.

As future work, we will consider the case of nonholomic and strong bracket generating distribution [20], where the system is controllable. This type of distributional system has many applications in automatic control, see for instance the paper [6], where the minimum wheel-rotation paths for differential-drive mobile robots are studied and the mathematical model is a driftless control affine system. It is interesting to use the PMP on the Lie algebroid, which in this case is the whole tangent space with the base given by vectors of distribution, together with the first iterated Lie brackets. 


\section{References}

[1] Agrachev, A.; Sachkov, Y. (2004). Control Theory from the Geometric Viewpoint. Encyclopedia of Mathematical Sciences 87, Springer, 2004.

[2] Brocket, R. (1973). Lie algebra and Lie groups in control theory. Geometrical Methods in Control Theory, Springer, 43-82, 1973.

[3] Caputo, M. (2005). Foundations of Dynamic Economic Analysis: Optimal Control Theory and Applications. Cambridge University Press, 2005.

[4] Caruntu, C. F.; Velandia-Cardenas, C.; Liu, X.; Vargas, A. (2018). Model predictive control of stochastic linear systems with probability constraints. Int. J. Comp. Commun. Control, 13(6), 927-937, 2018.

[5] Chazal, M.; Jouini, E.; Tahraoui, R. (2008). Production planning and inventories optimization: A backward approach in the convex storage cost case. J. Math. Economics, 44(9-10), 997-1023, 2008 .

[6] Chitsaz, H.; LaValle, S. M.; Balkcom, D.; Mason, M. (2009). Minimum Wheel-Rotation Paths for Differential-Drive Mobile Robots. The International Journal of Robotics Research, 28(1), 66-80, 2009 .

[7] Dubins, L.E. (1957). On curves of minimal length with a constraint on average curvature and with prescribed initial and terminal positions and tangents. American Journal of Mathematics, 79, 497-516, 1957.

[8] Feichtinger, G.; Hartl, R. (1985). Optimal pricing and production in an inventory model. European J. Operational Research, 19(1), 45-56, 1985.

[9] Gayon, J. P.; Vercraene, S.; Flapper, S. D. (2017). Optimal control of a production-inventory system with product returns and two disposal options. European J. Operational Research, 262(2), 499-508, 2017.

[10] Isidori, A. (1995). Nonlinear Control Systems. Springer, 1995.

[11] Jurdjevic, V. (2008). Geometric Control Theory. Cambridge Studies in Advanced Mathematics 52, Cambridge University Press, 2008.

[12] Kamien, M.I.; Schwartz, N.L. (2006). Dynamic optimization. Elsevier, 2006.

[13] LaValle, S. M. (2006). Planning Algorithms. Cambridge University Press, 2006.

[14] Liu, R.; Liu, G. (2018). Maximum principle for a nonlinear size-structured model of fish and fry management. Nonlinear Analysis: Modelling and Control, 23(4), 533-552, 2018.

[15] Maccini, L. J.; Moore, B.; Schaller, H. (2015). Inventory behavior with permanent sales stocks. J. Economic Dynamics and Control, 53, 290-313, 2015.

[16] Martinez, E. (2004). Reduction in optimal control theory. Rep. Math. Phys., 53(1), 79-90, 2004.

[17] Mackenzie, K. (1987). Lie Groupoids and Lie Algebroids in Differential Geometry. London Mathematical Society Lecture Note Series 124, Cambridge University Press, 1987.

[18] Olsson, F. (2019). Simple modeling techniques for base-stock inventory systems with state dependent demand rates. Mathematical Methods of Operations Research, 90, 61-76, 2019.

[19] Ortega, M.; Lin, L. (2004). Control theory applications to the production-inventory problem: a review. International Journal of Production Research, 42(11), 2303-2322, 2004. 
[20] Popescu, L. (2009). Lie algebroids framework for distributional systems. Annals Univ. Al. I. Cuza, Iasi, series I, Mathematics, 55(2), 257-274, 2009.

[21] Popescu, L. (2019). Applications of driftless control affine systems to a problem of inventory and production. Studies in Informatics and Control, 28(1), 25-34, 2019.

[22] Sethi, S. P.; Thompson, G. L. (2000). Optimal Control Theory: Applications to Management Science and Economics. Springer, 2000.

[23] Weber, T.A. (2011). Optimal Control Theory with Applications in Economics. MIT Press, 2011.

\section{(c) (1) (8)}

Copyright (C) 2020 by the authors. Licensee Agora University, Oradea, Romania.

This is an open access article distributed under the terms and conditions of the Creative Commons Attribution-NonCommercial 4.0 International License.

Journal's webpage: http://univagora.ro/jour/index.php/ijccc/

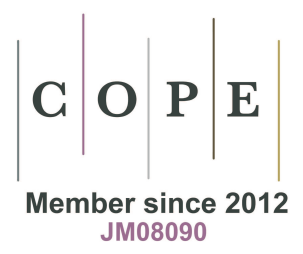

This journal is a member of, and subscribes to the principles of, the Committee on Publication Ethics (COPE).

https://publicationethics.org/members/international-journal-computers-communications-and-control

Cite this paper as:

Popescu, L.; Militaru, N.D.; Mituca, O.M.(2020). Optimal Control Applications in the Study of Production Management, International Journal of Computers Communications \& Control, 15(2), 3859, 2020.

https://doi.org/10.15837/ijccc.2020.2.3859 